\title{
Optimal Storage Allocation for Road Side Unit Aided Vehicular Mobile Content Dissemination
}

\author{
Wenyu Ren ${ }^{\dagger}$, Yong $\mathrm{Li}^{\dagger}$, Depeng Jin ${ }^{\dagger}$, Pan Hui ${ }^{\ddagger}$ \\ $\dagger$ Department of Electronic Engineering, Tsinghua University, Beijing 100084, China \\ $\ddagger$ Deutsche Telekom Laboratories/TU-Berlin, Ernst-Reuter-Platz 7, Berlin 10587 \\ yong-li07@mails.tsinghua.edu.cn
}

\begin{abstract}
Road Side Units (RSUs), which enable the vehicles to infrastructure communication, are deployed along the roadside to handle the growing communication demands as the number of vehicles increases. How to efficiently use these RSUs to enhance the vehicular network performance becomes an important problem after the optimal RSU placement schemes were proposed. In this poster, we investigate this problems by considering the application of vehicular mobile content dissemination. By collecting and analyzing the vehicular mobility traces from about 27,000 operational taxis during one month in Beijing city, we find that the contact patterns between the RSUs and vehicles obey exponential distribution. Based on this fact, We formulate the optimal mobile content dissemination problem with heterogeneous data items and limited storage as an integer linear programming, which can be solved by widely existing optimization suites like CPLEX and YALMIP.
\end{abstract}

\section{Categories and Subject Descriptors}

C.2.0 [Computer-Communication Networks]: General; C.4 [Performance of Systems]: [Modeling techniques, Performance attributes]

\section{Keywords}

Storage allocation, road side unit, content dissemination

\section{INTRODUCTION}

Recently, as more and more vehicles are equipped with devices to provide wireless communication capacities, interests on vehicular communications and networks have grown significantly [2]. Many consortia and standardization bodies are actively develop technologies and protocols for information transmission among vehicles and between the vehicles and infrastructure equipments, where exists fixed devices, i.e., Road Side Units (RSUs) deployed along the roadside.

Permission to make digital or hard copies of all or part of this work for personal or classroom use is granted without fee provided that copies are not made or distributed for profit or commercial advantage and that copies bear this notice and the full citation on the first page. To copy otherwise, to republish, to post on servers or to redistribute to lists, requires prior specific permission and/or a fee.

CoNEXT Student'12, December 10, 2012, Nice, France.

Copyright 2012 ACM 978-1-4503-1779-5/12/12 ...\$15.00.
Recent works focused on how to deployed RSU infrastructure to handle the growing communication demands as the number of vehicles increases and proposed optimal RSU placement schemes with the consideration of the vehicular traffic and city structures[1]. With the optimal RSU deployment,the left problem is that how to efficiently use the RSU to improve the network performance. In this poster, we investigate such problems by considering the application of vehicular mobile content dissemination. More specifically, we study the problem that how the system buffers the mobile content in the RSU aided mobile content sharing to enhance the dissemination efficiency. Our novel contribution is threefold which can be summarized as follows:

- We collect real mobility traces from about 27,000 operational taxis during one month in Beijing city, which records the key contact patterns between the RSU and vehicles of mobility in a large city, and enable us to analysis the problem in a realistic consideration.

- We consider the RSU-aided vehicular mobile content dissemination problem with the realistic heterogeneous settings that 1) the network contains heterogeneous vehicles in terms of data preference, 2) the mobile data items are multi-types of different delay sensitivity and size, and 3) the RSU' storages for content sharing are limited in size. These realistic conditions were not taken into account in the previous works for simplicity reasons.

- We formulate this optimal heterogeneous mobile content dissemination problem as a Integer Linear Programming (ILP) problem, which can be solved by widely existing optimization suites like CPLEX and YALMIP.

\section{TRACE COLLECTION AND CONTACT PATTERNS}

In collecting Beijing trace, we used the mobility track logs obtained from 27,000 participating Beijing taxis carrying GPS receivers during the whole month of May in 2010. Specifically, we utilized the GPS devices to collect the taxis locations and timestamps and GPRS modules to report the records every 15 seconds for moving taxis. The specific information contained in such a report includes: the taxi's ID, the longitude and latitude coordinates of the taxi's location, timestamps, instant speed and heading. Beijing trace is the largest vehicular data trace available.

After obtaining the traces, we assume the RSUs are placed in intersections of the main road, which is usually obtained 
by the current optimal placement algorithms, and investigate the patterns that how often the vehicles will enter into the communication coverage areas, defined as contact interval, and how long the vehicles will stay in the coverage areas, defined as contact duration. We extract the contacts from the trace, and investigate the complementary cumulative distribution function (CCDF) of the contact interval and duration and find that both the contact interval and duration obey exponential distribution.

\section{SYSTEM MODEL AND PROBLEM FOR- MULATION}

In the investigated system, there are $R+U$ nodes, where there are $R$ RSUs buffering the data and $U$ vehicles requesting the data. we use $\mathcal{R}$ and $\mathcal{U}$ to denote the set of RSUs and vehicles respectively, where $|\mathcal{R}|=R$ and $|\mathcal{U}|=U$. For any RSU $r \in \mathcal{R}$, let $L_{r}$ be the size of its buffer. Vehicles can communicate with the RSU only when they move into its coverage area, which is referred to as a communication contact. During the communication contact, nodes can transmit the mobile data in the rate of $v$ Bytes per second. Following the observation in the Beijing vehicular trace, we assume that the communication contact interval between RSU $r$ and vehicle $u$ obeys the Poisson process with contact rate $\theta_{u r}$, and the duration of their contact follows an exponential distribution with rate parameter $\lambda_{u r}$. During the contact, the transmitting rate remains $\eta$. Since in reality there are many different types of mobile data, for example, multimedia newspapers, weather forecasts, movie trailers, etc., we model the mobile traffics of $C$ different data items, labelled as $\mathcal{C}$. For any $c \in \mathcal{C}$, its data length is $l_{c}$. Using digital fountain codes, we generate $n_{c}=\frac{l_{c} \xi}{g}$ chunks from the message $c$, where $g$ is the size of each chunk and $\xi>1$. The original message $c$ can be reconstructed using any $v_{c}=\frac{(1+\varepsilon) l_{c}}{g}$ of the chunks, where $\varepsilon>0$. The lifetime for data $c$ is $\stackrel{g}{T_{c}}$. Finally, the interest probability of vehicles $u \in \mathcal{U}$ in mobile data $c \in \mathcal{C}$, defined by $\omega_{u, c}$. For any vehicles $u \in \mathcal{U}$, let $\omega_{u}=\left[\omega_{u, 1}, \omega_{u, 2}, \ldots, \omega_{u, C}\right]$ to be its affection to each of the data, whose sum is 1 .

For any $r \in \mathcal{R}$ and $u \in \mathcal{U}$, denote the number of chunks for data $c$ that $u$ receive from $r$ with $\pi_{u r c}$, where $0 \leq \pi_{u r c} \leq$ $v_{c}$. Assume in $c$ 's lifetime $T_{c}$, there are $N_{u r c}$ times of meet between $u$ and $r$ during which chunks of $c$ are transmitted. Then we have

$$
P\left(N_{u r c}=j\right)=\frac{e^{-\theta_{u r} \omega_{u c} T_{c}}\left(\theta_{u r} \omega_{u c} T_{c}\right)^{j}}{j !},(0 \leq j<+\infty) .
$$

Let $\tau_{u r c}(m)\left(1 \leq m \leq N_{u r c}\right)$ be the duration of each transmission of $c$. We have $\bar{\tau}_{u r c}(m) \sim \exp \left(\lambda_{u r}\right)$, which can also be written as $\tau_{\text {urc }}(m) \sim \Gamma\left(1, \frac{1}{\lambda_{u r}}\right)$. Define the total duration of $N_{u r c}$ times of transmission as $T\left(N_{u r c}\right)=\sum_{m=1}^{N_{u r c}} \tau_{u r c}(m)$. Вy the property of Gamma distribution, we have $T\left(N_{\text {urc }}\right) \sim$ $\Gamma\left(N_{u r c}, \frac{1}{\lambda_{u r}}\right)$. Then for $0 \leq i \leq v_{c}-1$,

$$
P\left(\pi_{u r c}=i \mid N_{u r c}=j\right)=\frac{\gamma\left(j, \frac{\lambda_{u r}(i+1) g}{\eta}\right)-\gamma\left(j, \frac{\lambda_{u r} i g}{\eta}\right)}{\Gamma(j)}
$$

where $\gamma(j, \lambda x)$ is the incomplete gamma function. For $i=$

$$
\begin{aligned}
& \quad P\left(\pi_{u r c}=v_{c} \mid N_{u r c}=j\right)=P\left(T\left(N_{u r c}\right) \geq \frac{v_{c} g}{\eta} \mid N_{u r c}=j\right) \\
& =1-F_{\Gamma}\left(\frac{v_{c} g}{\eta} ; j, \frac{1}{\lambda_{u r}}\right)=\frac{\Gamma(j)-\gamma\left(j, \frac{\lambda_{u r} v_{c} g}{\eta}\right)}{\Gamma(j)},
\end{aligned}
$$

From the law of total probability, we have

$$
\begin{gathered}
P\left(\pi_{u r c}=i\right)=\sum_{j=0}^{+\infty} P\left(N_{u r c}=j\right) P\left(\pi_{u r c}=i \mid N_{u r c}=j\right) \\
\left\{\begin{array}{c}
\sum_{j=0}^{+\infty} \frac{e^{-\theta u r \omega_{u c} T_{c}\left(\theta_{u r} \omega_{u c} T_{c}\right)^{j}}}{j !} \frac{\gamma\left(j, \frac{\lambda u r(i+1) g}{\eta}\right)-\gamma\left(j, \frac{\lambda u r i g}{\eta}\right)}{\Gamma(j)}, \\
\text { for } \quad 0 \leq i \leq v_{c}-1 \\
\sum_{j=0}^{+\infty} \frac{e^{-\theta u r \omega_{u c} T_{c}}\left(\theta_{u r} \omega_{u c} T_{c}\right)^{j}}{j !} \frac{\Gamma(j)-\gamma\left(j, \frac{\lambda u r v_{c} g}{\eta}\right)}{\Gamma(j)} \\
\text { for } i=v_{c}
\end{array}\right.
\end{gathered}
$$

Let $\Phi_{u c}=\left(\pi_{u 1 c}, \pi_{u 2 c}, \ldots, \pi_{u R \mid c}\right)$, where $0 \leq \pi_{u r c} \leq v_{c}$. For any $\phi_{u c} \in \Phi_{u c}$ where $\phi_{u c}=\left(i_{1}, i_{2}, \ldots, i_{R}\right)$, the probability of observing it is $P\left(\phi_{u c}\right)=\prod_{r \in \mathcal{R}} P\left(\pi_{u r c}=i_{r}\right)$

Define $x_{r c}$ as the number of chunks of message $c$ on $r$, $x_{r c} \in\left\{0,1,2, \ldots, v_{c}\right\}$. For each $\phi_{u c} \in \Phi_{u c}$, define $y_{u r c \phi}=$ $\min \left(\phi_{u c}(r), x_{r c}\right)$, where $1 \leq r \leq R$. Define $h_{u c \phi}=I\left(\sum_{r=1}^{R} y_{u r c \phi} \leq\right.$ $\left.v_{c}\right)$. The utility function can be written as

$$
U=\sum_{u \in \mathcal{U}} \sum_{c \in \mathcal{C}} \sum_{\phi_{u c} \in \Phi_{u c}} P\left(\phi_{u c}\right) h_{u c \phi}
$$

Further, the problem can be defined as an integer linear programming problem

$$
\begin{array}{r}
\text { Maximize } U \\
\text { s.t. } \quad v_{c} h_{u c \phi}-\sum_{r=1}^{R} y_{u r c \phi_{u c}} \leq 0, \\
y_{u r c \phi} \leq \phi_{u c}(r), y_{u r c \phi} \leq x_{r c}, \sum_{c \in \mathcal{C}} x_{r c} \leq L_{r}, \sum_{r \in \mathcal{R}} x_{r c} \leq n_{c}, \\
h_{u c \phi} \leq 1, x_{r c} \leq v_{c}, h_{u c \phi}, y_{u r c \phi}, x_{r c} \geq 0 .
\end{array}
$$

\section{SOLUTION AND DISCUSSION}

As stated above, we formulate our problem into a integer linear programming problem, which can be solved by widely existing optimization suite like CPLEX and YALMIP. As the solution space for this problems is large, it is relatively hard to deploy this method in real-time storage allocation. However, the solution of our linear programming problem is the optimal storage allocation and the value of the utility function can be seen as a theoretical upper bound, which is useful when we evaluate applicable algorithms such as greedy and heuristic ones.

\section{REFERENCES}

[1] A. Abdrabou and W. Zhuang. Probabilistic delay control and road side unit placement for vehicular ad hoc networks with disrupted connectivity. IEEE Journal on Selected Areas in Communications, 29(1):129-139, 2011.

[2] M. Khabazian, S. Aissa, and M. Mehmet-Ali. Performance modeling of message dissemination in vehicular ad hoc networks with priority. IEEE Journal on Selected Areas in Communications, 29(1):61-71, 2011. 